\title{
Special issue on the 12th international conference on computational management science
}

\author{
Miloš Kopa' ${ }^{1}$ Wolfram Wiesemann²
}

Published online: 10 January 2017

(C) Springer-Verlag Berlin Heidelberg 2017

This issue is based on presentations at the twelfth International Conference on Computational Management Science held at Charles University, Prague, May 27-29, 2015. The topics covered highlight the role played by computational techniques to solve relevant real-world problems in management science.

The CMS conference is an annual meeting associated with the Journal of Computational Management Science. The aim of this conference is to provide a forum for theoreticians and practitioners from academia and industry to exchange knowledge, ideas and results in a broad range of topics relevant to the theory and practice of computational methods, models and empirical analysis for decision making in economics, engineering, finance and management. The focus is on all computational aspects of management science: theoretical and empirical studies of computational methods, models and empirical analysis. These include computational economics, finance and statistics, energy, scheduling, supply chains, design, analysis and applications of optimisation algorithms, deterministic, dynamic, stochastic, robust and combinatorial optimisation models, solution algorithms, learning and forecasting such as neural networks and genetic algorithms, models and tools of knowledge acquisition, such as data mining, and all other topics in management science with the emphasis on computational paradigms.

Miloš Kopa

kopa@karlin.mff.cuni.cz

Wolfram Wiesemann

ww@imperial.ac.uk

1 Department of Probability and Mathematical Statistics, Sokolovská 83, Faculty of Mathematics and Physics, Charles University, Prague, Czech Republic

2 Imperial College Business School, South Kensington Campus, London SW7 2AZ, UK 
10.1007/s10287-016-0272-3, Maggioni, Potra and Bertocchi present and compare two popular paradigms for decision-making under uncertainty-stochastic programming and robust optimization-in the context of a supply chain optimization problem. The authors find that while providing comprehensive guarantees, single-stage robust optimization models can result in overly conservative solutions. This can in part be compensated by employing two-stage robust optimization models using affine decision rules.

10.1007/s10287-016-0253-6, de Ruiter, Brekelmans, Ben-Tal and den Hertog propose a novel robust optimization approach for production-inventory problems with uncertain customer demands. The authors argue that the input data of such problems (in particular, previously observed customer demands) is often inaccurate, and optimal decisions in view of the inaccurate data may lead to poor performance. Instead, the authors propose an extension of the adjustable robust optimization framework that treats the observed data itself as uncertain and that optimizes in view of the worst realization of both the future customer demands as well as the past data inaccuracies. The authors demonstrate the promising performance of their scheme in numerical experiments.

10.1007/s10287-016-0261-6, authored by Dupačová and Kozmík, proposes a new preprocessing technique for stochastic dual dynamic programs (SDDPs) that can significantly reduce the computation times. Their scheme is based on quantitative stability results, and it performs a stage-wise backward reduction of individual scenarios applied to a fixed branching structure of the scenario tree. The authors present computational results in which their technique results in substantial reductions of the runtimes of SDDP.

10.1007/s10287-016-0268-z, Szabó studies the Goldbach conjecture. The Goldbach conjecture goes back to a conversation between Goldbach and Euler in 1742, and it is one of the oldest and best-known unsolved problems in number theory. It claims that every even integer greater than 2 can be written as the sum of two primes. The author formulates an equivalent max-algebraic claim, which allows to examine the conjecture by the methods of max-algebras. By doing so, he sheds new light on this classical problem.

10.1007/s10287-016-0259-0, Krivulin proposes a new class of tropical optimization problems, which minimize a nonlinear objective function defined on vectors over an idempotent semifield subject to a set of inequality constraints. The author provides a complete solution to this problem by solving a parameterized vector inequality. The author also demonstrates the relevance of the problem class by applying it to a well-known time-constrained project scheduling problem.

10.1007/s10287-016-0265-2, authored by van Hulst, den Hertog and Nuijten, applies the robust optimization paradigm to a shift generation problem in workforce planning. Shift generation problems often need to be solved under significant uncertainty as the workload predictions are subject to change. By using an adverserial approach, the authors propose a model that generates robust shifts which are immunized against the uncertainty in the workload predictions. They also provide numerical results which show the value of their approach.

10.1007/s10287-016-0263-4, Vitali, Moriggia and Kopa study the problem of a private pension plan sponsor who wishes to determine the best pension funds for its 
members. The authors develop a multi-stage stochastic programming formulation with a multi-criteria objective function that (i) minimizes the Average Value at Risk Deviation of the final wealth, (ii) satisfies a wealth target in the final stage and (iii) satisfies other constraints regarding pension plan regulations. Numerical results illustrate the optimal dynamic portfolios and how they depend on the investors' preferences.

10.1007/s10287-016-0262-5, Branda and Hájek study the operational fixed interval scheduling problem, which assigns jobs with known start and completion times to a given set of machines so as to maximize the overall revenues. While the classical fixed interval scheduling problems are deterministic, practical problems are often characterized by random delays in the job processing times. The authors formulate two stochastic programming formulations to address such random delays. The resulting integer linear reformulations have the structure of flow problems and can therefore be solved more efficiently than comparable models from the literature.

The submissions clearly demonstrate the increasing importance of stochastic and robust decision-making. Due to the high complexity surrounding quantitative models, research on computational management science is likely to be of increasing significance in the future. Unfortunately, two of the authors passed away during the reviewing process: Jitka Dupačová (January 4, 2016) and Marida Bertocchi (November 16, 2016).

Jitka Dupačová was Professor at the Department of Probability and Mathematical Statistics, Faculty of Mathematics and Physics, Charles University in Prague (Czech Republic). She was an active member of various international and national scientific societies such as the Mathematical Optimization Society, Stochastic Programming Community, EURO WGs on Commodity and Financial Modeling and on Stochastic Optimization, and the Czech Econometric Society. She reached wide recognition for her results in stochastic programming and related areas of statistics and optimization, which is also the subject of her books and more than 150 published scientific papers. She is considered as one of the founders of stochastic programming. In consideration of her merits in the development of stochastic programming she was awarded one of twelve memorial plaques during the X. Symposium on Stochastic Programming (2004). She was a founder and chair of the PhD program on Econometrics and Operations Research at Charles University for more than 30 years.

Marida Bertocchi was Professor in Financial Mathematics at the Department of Management, Economics and Quantitative Methods of University of Bergamo (Italy) where she covered several institutional duties: among them Dean of the Faculty of Economics and Business Administration from November 1996 to October 2002, Director of the Department of Mathematics, Statistics, Computer Science and Applications from 2009 to 2012 and since 1992 Coordinator of the PhD Program in "Computational Methods for Economic and Financial Forecasting and Decision Making" (now "Analytics for Economics and Business"). She was an active member of various international and national scientific societies such as the Mathematical Optimization Society (MOS), Stochastic Programming Community (COSP), EURO WGs on Commodity and Financial Modeling and on Stochastic Optimizaton, European Financial Association (EFA). Her scientific expertise covers the fields of optimization, linear algebra, parallel computing and stochastic programming with applications to finance, insurance, energy and logistic sectors. She published more than 99 scientific papers and edited eight books. She participated in many applied scientific projects. 
Jitka and Marida were cooperating for more than 25 years in many joint papers and projects. They both are missed by the whole stochastic programming community. The issue is dedicated to their memory. 